\title{
Conhecimento de enfermeiras sobre a ocorrência de incidentes com pessoas idosas hospitalizadas
}

\author{
Awareness of nurses on the occurrence of incidents with \\ elderly hospitalized patients
}

\author{
Ludmila Anjos de Jesus' ${ }^{1}$, Maria Olívia Sobral Fraga de Medeiros², Mary Gomes Silva ${ }^{3}$ \\ ${ }^{1}$ Autora para correspondência. Escola Bahiana de Medicina e Saúde Pública. Salvador, Bahia, Brasil. \\ ORCID: 0000-0003-4956-4096. ludmila.anjos27@gmail.com \\ 2Escola Bahiana de Medicina e Saúde Pública. Salvador, Bahia, Brasil. ORCID: 0000-0001-6811-378X. mariamedeiros@bahiana.edu.br \\ ${ }^{3}$ Escola Bahiana de Medicina e Saúde Pública. Salvador, Bahia, Brasil. ORCID: 0000-0001-9145-868X. mgsilva@bahiana.edu.br
}

\begin{abstract}
RESUMO | OBJETIVO: Analisar o conhecimento de enfermeiras sobre a ocorrência de incidentes durante a assistência de enfermagem ao idoso hospitalizado. MÉTODOS: Estudo quantitativo-descritivo, realizado com enfermeiras assistenciais em unidades de internação de um centro geriátrico em Salvador-BA. RESULTADOS: Os incidentes mais citados pelas enfermeiras foram lesão por pressão $(26,15 \%)$ e quedas $(16,92 \%)$. Entre os principais fatores relacionados aos incidentes citados pelas profissionais destacaram-se as condições clínicas dos pacientes, contudo também foram apontados fatores institucionais e humanos. Sobre as medidas que devem ser adotadas após incidentes, $48 \%$ evidenciou que devem ser realizadas medidas educativas com a equipe e $32 \%$ correção dos fatores relacionados. CONCLUSÃO: Constatouse que as enfermeiras têm conhecimento sobre a ocorrência de incidentes com idosos na prática hospitalar. É fundamental sensibilizar os profissionais quanto à importância da capacitação teórico-prática. Compreender a segurança do paciente é imprescindível para o desenvolvimento de estratégias voltadas para minimizar os incidentes.
\end{abstract}

PALAVRAS-CHAVE: Segurança do paciente. Idoso. Enfermagem.

\begin{abstract}
OBJECTIVES: to analyze the awareness of nurses in relation to the occurrence of incidents during nursing care of hospitalized elderly patients. METHODS: A quantitativedescriptive study performed with registered nurses in a patient care unit of a geriatric center in Salvador-BA. RESULTS: The most mentioned incidents by the nurses were pressure ulcers $(26,15 \%)$ and fall injuries $(16,92 \%)$. Among the main factors related to the incidents mentioned by the professionals are the clinical conditions of patients; however institutional and human factors were also stated. Regarding measures to be adopted after the incidents, $48 \%$ emphasized that educational measures should be performed with the team and $32 \%$ highlighted the correction of the corresponding factors. CONCLUSION: It was observed that the nurses are aware of the occurrence of incidents with elderly patients during the practice. It is of elementary importance to raise awareness of professionals as to the importance of theoretical-practical training. Awareness of the safety of patients is critical for the development of strategies guided towards reducing such incidents.
\end{abstract}

kEYWORDS: Patient safety. Elderly. Nursing. 


\section{Introdução}

A segurança do paciente é definida como a redução, a um mínimo aceitável, do risco de dano desnecessário relacionado ao cuidado à saúde ${ }^{1}$. Tal temática que não é recente, tornou-se evidência mundial com a publicação do relatório To err is human pelo Instituto de Medicina do Estado Unidos, em 2000, por apontar que o número de mortes relacionadas aos erros durante a assistência superou as mortes causadas por acidentes automobilísticos, câncer de mama e síndrome da imunodeficiência adquirida, com consequente aumento dos custos sociais e econômicos².

A Organização Mundial de Saúde (OMS) objetivando o desenvolvimento de práticas e políticas de segurança em nível mundial criou, em 2004, a Aliança Mundial para Segurança do Paciente ${ }^{3}$. No Brasil, em 2008, criou-se a Rede Brasileira de Enfermagem e Segurança do Paciente (REBRAENSP), cujo propósito foi fortalecer a assistência de enfermagem segura e com qualidade ${ }^{4}$. E em 2013, o Ministério da Saúde instituiu o Programa Nacional de Segurança do Paciente (PNSP), que tem como finalidade o apoio e a promoção de iniciativas voltadas para a segurança' ${ }^{1}$.

O incidente é definido como um evento que poderia ter gerado ou gerou um dano desnecessário ao paciente ${ }^{1}$. O near miss, conhecido como quase erro, é o incidente que não atingiu o paciente ${ }^{1}$. Já o evento adverso é um incidente que resultou em um dano'.

No Brasil, a proporção de pessoas maiores de 60 anos se duplicará entre 2000 e 2050, passando a 22\% da população total ${ }^{5}$, o que é uma situação desafiadora devido a maior exigência de cuidados à saúde frente à fragilidade da estrutura das redes de atenção. As alterações biopsicossociais do envelhecimento quando associadas a presença de doenças crônicas e seus agravos favorecem a hospitalização aumentando o risco de ocorrência de eventos indesejáveis ${ }^{6}$.

Dessa forma, a hospitalização eleva a vulnerabilidade para ocorrência de eventos adversos devido a predisposição dos idosos a uma recuperação mais lenta, o que favorece um internamento prolongado, além da ocorrência de readmissões, que são acentuadas pela inabilidade dos profissionais em avaliar os aspectos primordiais da senescência e senilidade7. Assim, os idosos são os principais afetados por incidentes durante a prestação de cuidados.

Um estudo realizado em idosos internados em uma unidade de clínica cirúrgica de um hospital de ensino da região Centro-oeste Brasileira em 2017 evidenciou uma prevalência de $58,8 \%$ de eventos adversos nos idosos hospitalizados, com uma média de 2,04 eventos por internação ${ }^{8}$. Erros de medicação, quedas, retiradas não programas de dispositivos terapêuticos e lesões por pressão são os principais incidentes identificados e estão intimamente relacionados aos cuidados de enfermagem ${ }^{9}$.

Considerando que as enfermeiras realizam grande parte das ações de cuidado, elas ocupam uma posição crucial para promover a segurança do paciente idoso e realização do gerenciamento de riscos, já que estão intrinsecamente envolvidas na assistência, e compõem um expressivo número dentre os profissionais do hospital ${ }^{10}$. Para elas, conhecer o contexto da ocorrência de incidentes, bem como, os fatores de risco, contribuirá diretamente para a criação de estratégias voltadas para prevenir erros e assegurar a promoção da segurança $a^{10,11}$.

Neste âmbito, partindo-se do pressuposto que a ocorrência de incidentes durante a assistência de enfermagem compromete a saúde dos pacientes, podendo causar consequências irreversíveis, esta pesquisa tem como questão norteadora: qual o conhecimento das enfermeiras sobre os incidentes que ocorrem durante a assistência de enfermagem ao paciente idoso?

Considerando a relevância do tema proposto, o crescente envelhecimento populacional e suas repercussões biopsicossociais, e da carência de estudos na área ${ }^{4}$, essa pesquisa tem por objetivo geral analisar o conhecimento de enfermeiras sobre a ocorrência de incidentes durante a assistência de enfermagem ao idoso hospitalizado, e por objetivos específicos caracterizar o perfil profissional das enfermeiras relacionando-o com a ocorrência de incidentes assistenciais e conhecer os fatores relacionados à ocorrência de incidentes durante a assistência de enfermagem ao paciente idoso. 


\section{Metodologia}

Estudo de caráter quantitativo-descritivo, realizado nas unidades de internação de um centro de referência em geriatria e gerontologia filantrópico no município de Salvador-BA, no período de novembro a dezembro de 2017. Esse centro possui duas unidades de internação cada uma contendo 72 leitos (total de 144 leitos).

O centro geriátrico atende pacientes de perfis variados, com predominância de idosos com doenças crônicas não transmissíveis agudizadas, em tratamento de lesões crônicas, em investigação diagnóstica, com demências e neoplasias, em cuidados paliativos, e idosos em reabilitação, o que favorece o tempo de internamento prolongado.

A população do estudo foi composta por todas as enfermeiras que atuavam no centro geriátrico (total de 25 profissionais) que tiveram como critério de inclusão um tempo de atuação mínimo de 06 meses no referido centro. Dessa forma, 8 foram excluídas por se encontrarem de férias, licença maternidade e por atuarem na administração ou gerência das unidades. Assim, 17 enfermeiras participaram do estudo.

A coleta de dados consistiu na utilização de um questionário auto-aplicável e estruturado elaborado pelas autoras a partir dos objetivos dessa pesquisa, com base na leitura de materiais científicos. O questionário continha 13 perguntas objetivas, sendo dividido em duas partes, a primeira contendo perguntas para caracterização dos sujeitos, e a segunda contendo questões da pesquisa, na qual mais de alternativa poderia ser marcada.

O questionário foi respondido conforme a disponibilidade do profissional no seu ambiente de trabalho, após a entrega da carta-convite, esclarecimentos sobre o sigilo e anonimato, assinatura do Termo de Consentimento Livre e Esclarecido, e sem a presença do pesquisador. Posteriormente foram depositados em uma pasta sem a identificação.

Os dados foram quantificados e tabulados no programa Excel Microsoft versão 2010. Foi utilizada a estatística descritiva para a caracterização das variáveis, com uso de frequência absoluta e porcentagens.

Para realização desse estudo foram respeitadas as normas brasileiras para pesquisas com seres humanos recomendados pela Resolução n 466/2012 do Conselho Nacional de Saúde, sendo o projeto aprovado por um Comitê de Ética em Pesquisa através da CAAE n77787317.4.0000.0047.

\section{Resultados}

A amostra foi composta por 17 enfermeiras, que apesar do tempo de atuação (47,05\% das enfermeiras atuam no referido centro de 2 a 5 anos) apenas 11,76\% possuem especialização na área de geriatria/gerontologia. Contudo, $82,35 \%$ delas afirmaram terem participado de cursos específicos na área nos últimos 2 anos. A tabela 1 representa a caracterização dos participantes do estudo. 


\begin{tabular}{|c|c|c|c|}
\hline & & $\mathbf{n}$ & $\%$ \\
\hline \multirow[t]{4}{*}{ Sexo } & Masculino & 0 & - \\
\hline & Feminino & 17 & 100 \\
\hline & $<2$ anos & 1 & 5,88 \\
\hline & de 2 a 5 anos & 8 & 47,05 \\
\hline \multirow[t]{3}{*}{ Tempo de formação } & de 5 a 10 anos & 7 & 41,17 \\
\hline & de 10 a 15 anos & 0 & - \\
\hline & $>15$ anos & 1 & 5,88 \\
\hline \multirow{3}{*}{ Especialização em geriatria/gerontologia } & Sim & 2 & 11,76 \\
\hline & Não & 13 & 76,47 \\
\hline & Cursando & 2 & 11,76 \\
\hline \multirow{3}{*}{ Especialização em outra área } & Sim & 13 & 76,47 \\
\hline & Não & 2 & 11,76 \\
\hline & Cursando & 2 & 11,76 \\
\hline \multirow{5}{*}{ Anos de atuação no Centro Geriátrico } & $>6$ meses e $<2$ anos & 2 & 11,76 \\
\hline & de 2 a 5 anos & 8 & 47,05 \\
\hline & de 5 a 10 anos & 7 & 41,17 \\
\hline & de 10 a 15 anos & 0 & - \\
\hline & $>15$ anos & 0 & - \\
\hline \multirow{2}{*}{$\begin{array}{l}\text { Participação em cursos ou treinamento } \\
\text { específicos nas áreas de } \\
\text { geriatria/gerontologia nos últimos } 2 \text { anos }\end{array}$} & Sim & 14 & 82,35 \\
\hline & Não & 3 & 17,64 \\
\hline \multirow{2}{*}{$\begin{array}{l}\text { Profissionais com outros vínculos } \\
\text { empregatícios }\end{array}$} & Sim & 8 & 47,05 \\
\hline & Não & 9 & 52,84 \\
\hline \multirow{4}{*}{ Carga horária de trabalho semanal } & $\leq 36$ horas & 7 & 41,17 \\
\hline & de 36 a 44 horas & 3 & 17,64 \\
\hline & de 45 a 72 & 3 & 17,64 \\
\hline & $>72$ horas & 4 & 23,52 \\
\hline
\end{tabular}

$\mathrm{N}=$ número absoluto de respostas

$\%=$ porcentagem 
Em relação aos incidentes mais frequentes vivenciados pelas profissionais, há um destaque para as lesões por pressão e quedas, conforme evidencia o gráfico 1.

Gráfico 1. Incidentes que ocorrem com mais frequencia durante aprestação de cuidados de enfermagem ao paciente idoso em unidades de internação de um centro geriátrico, Salvador, Bahia, Brasil, 2018

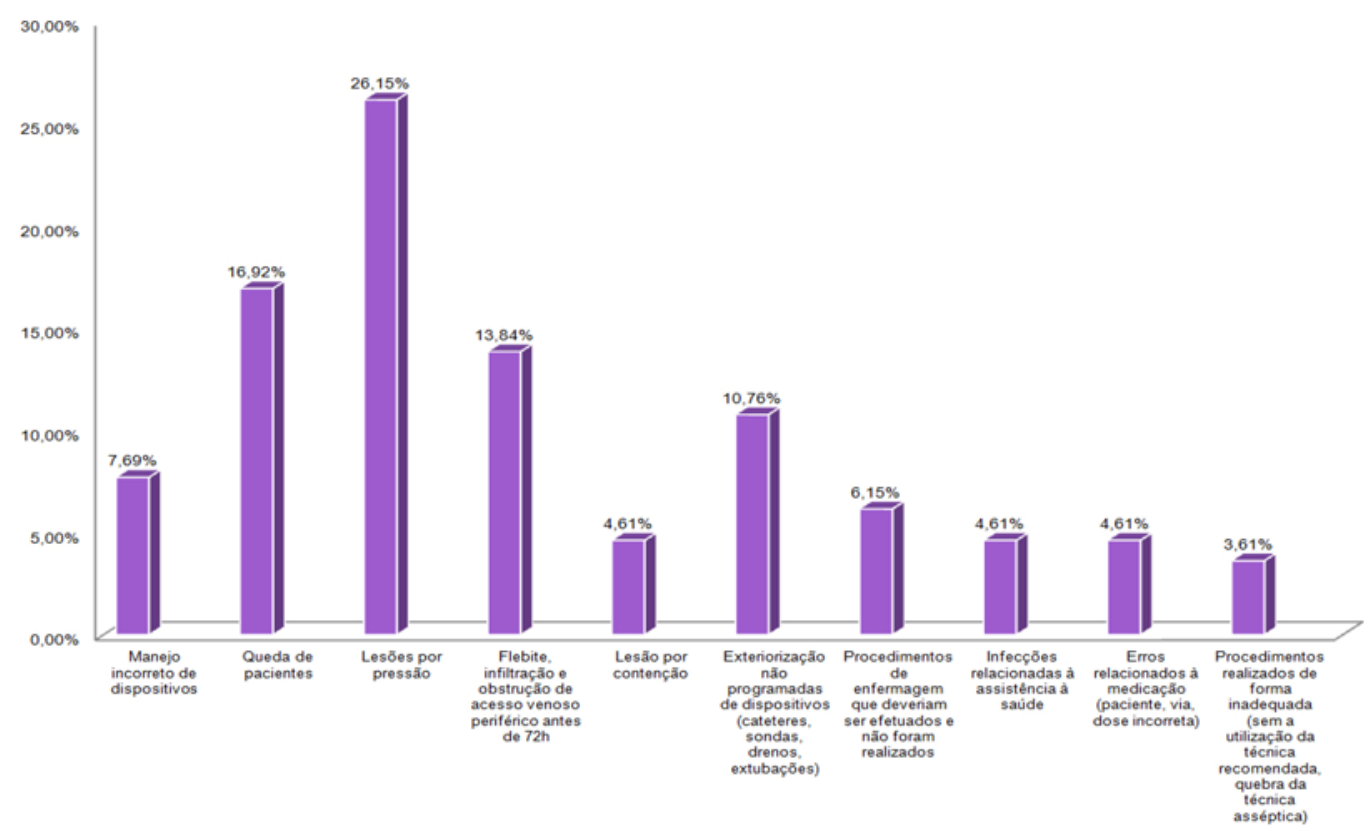

Quanto aos fatores relacionados aos incidentes, 49,35\% das respostas estavam relacionadas às condições clínicas dos pacientes, $25,97 \%$ a questões institucionais e $24,67 \%$ a fatores humanos. A tabela 2 mostra os principais fatores relacionados à ocorrência de incidentes em idosos.

Tabela 2. Principais fatores relacionados à ocorrência de incidentes durante a assistência de enfermagem ao paciente idoso, Salvador, Bahia, Brasil, 2018 (continua)

\begin{tabular}{cccc}
\hline & & $\mathbf{n}$ & $\mathbf{\%}$ \\
\hline & Imobilidade & 17 & 22,36 \\
& Incapacidade de Comunicação & 7 & 9,21 \\
Condições Clínicas & Incapacidade cognitiva & 14 & 18,42 \\
& Incontinências & 14 & 18,42 \\
& Fragilidade & 15 & 19,73 \\
& Déficit nutricional & 9 & 11,84 \\
& Outros & 0 & - \\
\hline
\end{tabular}




\begin{tabular}{|c|c|c|c|}
\hline & & $\mathbf{n}$ & $\%$ \\
\hline \multirow{12}{*}{ Fatores institucionais } & $\begin{array}{l}\text { Ambiente físico (barulho, agitação, } \\
\text { estímulos visuais e calor) }\end{array}$ & 8 & 20,00 \\
\hline & Condições de trabalho insalubres & 3 & 7,50 \\
\hline & $\begin{array}{l}\text { Delegação de tarefas sem supervisão } \\
\text { adequada }\end{array}$ & 7 & 17,50 \\
\hline & Ausência de protocolos e rotinas & 1 & 2,50 \\
\hline & $\begin{array}{c}\text { Falta de padronização da nomenclatura } \\
\text { dos medicamentos e o uso de } \\
\text { abreviaturas }\end{array}$ & 2 & 5,00 \\
\hline & $\begin{array}{c}\text { Inadequação de recursos materiais e } \\
\text { equipamentos }\end{array}$ & 2 & 5,00 \\
\hline & $\begin{array}{l}\text { Deficiências de formação, treinamento e } \\
\text { atualização }\end{array}$ & 4 & 10,00 \\
\hline & $\begin{array}{l}\text { Número inadequado de recursos } \\
\text { humanos }\end{array}$ & 5 & 12,50 \\
\hline & $\begin{array}{l}\text { Trabalho em equipe deficiente (falhas na } \\
\text { comunicação) }\end{array}$ & 5 & 12,50 \\
\hline & $\begin{array}{c}\text { Distanciamento de ações próprias da } \\
\text { enfermagem }\end{array}$ & 2 & 5,00 \\
\hline & $\begin{array}{l}\text { Ausência e/ou treinamento insuficiente } \\
\text { para manipulação dos equipamentos, } \\
\text { artefatos diagnósticos e terapêuticos }\end{array}$ & 1 & 2,5 \\
\hline & Outros & 0 & - \\
\hline \multirow{8}{*}{ Fatores Humanos } & Sobrecarga de serviço & 7 & 18,42 \\
\hline & Desmotivação & 4 & 10,52 \\
\hline & Falta de atenção & 15 & 39,47 \\
\hline & Estresse & 4 & 10,52 \\
\hline & $\begin{array}{c}\text { Má interpretação de prescrições } \\
\text { (ortografia de má qualidade, erros nas } \\
\text { prescrições...) }\end{array}$ & 4 & 10,52 \\
\hline & Fadiga & 1 & 2,63 \\
\hline & Dobra do serviço & 1 & 2,63 \\
\hline & Outros (Falta de conhecimento) & 2 & 5,26 \\
\hline
\end{tabular}

$\mathrm{N}=$ número absoluto de respostas

$\%=$ porcentagem 
Sobre o que acontece administrativamente após a ocorrência de incidentes, $44 \%$ afirmaram registro em impresso específico, 36\% registro em prontuário, e $4 \%$ apenas comunicação oral, sendo que, $16 \%$ apontaram outros (conversa com o profissional (4\%), advertência verbal ou escrita (8\%), e registro em prontuário associado ao registro em impresso específico e comunicação oral (4\%).

Entre as medidas que devem ser adotadas após a ocorrência de incidentes, $48 \%$ das respostas evidenciaram medidas educativas, $32 \%$ correção dos fatores relacionados, e $20 \%$ medidas punitivas, sendo que nenhum enfermeiro apontou medidas jurídicas ou legais.

Considerando as estratégias que devem ser utilizadas para evitar a ocorrência de incidentes, 31,42\% das respostas evidenciaram investimentos em capacitação e treinamento, $28,57 \%$ implantação de um sistema de detecção, correção e prevenção de erros (cultura de segurança), 20\% melhor comunicação entre a equipe (prática colaborativa), e $20 \%$ estímulo à notificação dos erros.

\section{Discussão}

Todas as participantes eram do sexo feminino, o que ratifica a enfermagem como uma profissão predominantemente feminina devido ao contexto histórico de seu surgimento, também verificado em outro estudo ${ }^{12}$.

A maioria das profissionais tinha entre 2 a 5 anos de formação. É possível inferir que enfermeiras com pouca experiência consistem em um fator predisponente para a ocorrência de incidentes, contudo, enfermeiras mais experientes podem cometer erros por excesso de confiança ${ }^{13}$. Todavia, não é possível desvincular a experiência do julgamento clínico, pois a primeira confere maior agilidade e assertividade nas ações de enfermagem.

Associando tempo de experiência com eventos adversos, um estudo internacional realizado em 2009 demonstrou que o número de anos de experiência das enfermeiras foi inversamente relacionado com a ocorrência de infecções do trato urinário em pacientes.13 Entretanto, tempo de experiência desassociados de atualização reproduzem em uma prática obsoleta, ratificando que a busca periódica pelo aperfeiçoamento é ideal.
Em relação ao tempo de atuação no centro geriátrico $47,05 \%$ das enfermeiras atuam na unidade de 2 a 5 anos e $41,17 \%$ de 5 a 10 anos, e nenhuma há mais de 10 anos. Contudo, apesar do tempo de atuação, $76,47 \%$ das enfermeiras não possuiam especialização em nas áreas de geriatria ou gerontologia. Mas, 70,58\% eram especialistas em outra área do conhecimento. No entanto, há uma predominância de participação em cursos específicos na área nos últimos 2 anos, o que é corroborado pelo incentivo ofertado pelo grupo de educação permanente da instituição.

Apesar de não existir regulamentação que exija especialização para atuação na área, isso proporcionaria um maior subsídio técnico-científico e refletiria numa melhor assistência prestada ao paciente. Estudo internacional prévio, realizado em unidades de terapia intensiva, verificou que a taxa de quedas de pacientes foi inversamente relacionada à proporção de enfermeiras com especialização ${ }^{13}$. Ratificando, portanto, que o aprimoramento técnico possui uma relação diretamente proporcional com a segurança do paciente.

Em relação a possuir outros vínculos empregatícios, $52,94 \%$ das enfermeiras negaram. No âmbito da carga horária de trabalho semanal, a maioria das enfermeiras afirmaram trabalharem com uma carga horária igual ou inferior a 36 horas/semana, e apenas $23,52 \%$ declaram trabalhar mais de 72 horas/semana. Na perspectiva da segurança, esse resultado é favorável, visto que, uma pesquisa evidenciou que o risco de cometer erros foi significativamente maior quando os turnos de trabalho eram superiores a doze horas ou quando as profissionais trabalhavam mais de 40 horas por semana ${ }^{14}$. Sugere-se, portanto, que existe uma relação negativa entre o aumento do número de horas trabalhadas e o nível da assistência prestada.

Entre os principais incidentes citados pelas enfermeiras que ocorrem no idoso hospitalizado foram identificadas lesões por pressão $(26,15 \%)$, queda de pacientes $(16,92 \%)$, e flebite, infiltração e obstrução de acesso venoso periférico antes de $72 \mathrm{~h}$ (13,84\%), de maneira análoga a um estudo realizado em um hospital universitário no estado de São Paulo, o qual tinha por objetivo de identificar as principais iatrogenias ocorridas com idosos internados ${ }^{15}$. 
A ocorrência de lesões por pressão é multifatorial, mas os pacientes idosos apresentam vulnerabilidade para esse incidente devido à fragilidade cutânea e capilar, desnutrição, redução da sensibilidade, presença de comorbidades, maior período de internação e mobilidade física prejudicada ${ }^{16}$. Apesar da relação com as condições clínicas do idoso, a ocorrência dessas lesões também é resultado da qualidade da assistência prestada ${ }^{15}$.

A indicação das participantes sobre a ocorrência de quedas foi significativa. Os prejuízos relacionados a esse incidente, além de físicos, como traumatismos e fraturas, podem ser também financeiros, como o prolongamento do tempo de internação e a necessidade de novas intervenções, afetando a independência e a capacidade funcional dos indivíduos. Entre os motivos associados a quedas, destacam-se as condições clínicas dos pacientes, como o rebaixamento do nível de consciência, incontinência, alterações visuais e períodos de agitação psicomotora, além de falhas na vigilância desses pacientes ${ }^{17}$.

Em uma proposta de gestão de cuidados, a Rede Brasileira de Enfermagem e Segurança do Paciente, recomenda o estabelecimento de um programa de avaliação periódica dos riscos para quedas e lesões por pressão, com a utilização de escalas como, a Morse Fall Scale e Escala de Braden 4 .

A redução do número de quedas e da ocorrência de lesões por pressão está entre as metas da segurança do paciente, com protocolos básicos divulgados e implantados na instituição, o que pode ter contribuído para a prevalência da citação pelas enfermeiras ${ }^{18}$.

Flebite, infiltração e obstrução de acesso venoso periférico antes de $72 \mathrm{~h}$ também foram apontados, e entre as principais causas destacam-se o calibre do dispositivo, a fragilidade capilar e túnica íntima do vaso com maior tendência a sofrer processos inflamatórios presente em idosos, e pelas propriedades dos medicamentos e da infusão. Ademais, os idosos muitas vezes apresentam grande número de comorbidades, acarretando em agravamento do estado de saúde e maior número de drogas utilizadas, repercutindo em piora da fragilidade na rede vascular ${ }^{19}$. O tipo e a forma de fixação do cateter periférico também estão relacionados, o que acarreta aumento no número de punções, potencializando os danos na rede venosa periférica, maior risco de infecção, dor e desconforto ${ }^{20}$.
Um aspecto notável evidenciado foi que infecções, erros relacionados à medicação, lesão por contenção e procedimentos realizados de forma inadequada obtiveram as menores porcentagens. Dado que contrasta com a literatura ${ }^{9,15}$, podendo sugerir que há falta de conhecimento e subnotificação quanto a esses incidentes.

Vários elementos podem predispor a ocorrência de um incidente, entre eles estão os elementos individuais do paciente, deficiências da educação e treinamento, condições de trabalho, falhas na comunicação e questões organizacionais ${ }^{21}$. Neste estudo houve uma predominância de fatores relacionados a condições clínicas do idoso com destaque para a imobilidade e a fragilidade. A imobilidade e fragilidade na pessoa idosa são indicadores do declínio da funcionalidade, perda da capacidade funcional, com prejuízo significativo da autonomia e independência, o que torna o sujeito mais vulnerável e com maior dependência ao cuidado.

Entre os fatores institucionais, o ambiente físico, a delegação de tarefas sem supervisão, número inadequado de recursos humanos e trabalho em equipe deficiente foram prevalentes. Apenas 10\% das enfermeiras apontaram deficiências de formação profissional como fator relacionado aos incidentes. A ausência e/ou treinamento insuficiente, e ausência de protocolos obtiveram as menores porcentagens, sendo possível desvelar que as enfermeiras não compreendem a formação e padronização como parte fundamental da cultura de segurança.

Considerando que as condições de trabalho interferem diretamente na qualidade do cuidado, 10 os fatores institucionais observados justificam os incidentes elencados.

Fatores humanos, como a falta de atenção e a sobrecarga de serviço também foram citados. Há uma relação direta entre esses dois pontos, e quando associados favorecem ao aumento da chance de ocorrer um evento adverso em até 2 vezes ${ }^{22}$. Contudo, é primordial assegurar que as condutas técnicas desse profissional não estejam sob interferência direta da síndrome de Bournout.

O modelo mais utilizado para a análise dos erros foi o proposto por James Reason, em 1990, denominado Modelo do Queijo Suíço. De acordo com essa teoria, 
os erros devem passar por várias camadas de proteção, as fatias do queijo, para resultar em um prejuízo. Assim, o erro não deve ser visto com uma abordagem isolada, mas sim, como o resultado de uma sequência alinhada de falhas².

À luz desse modelo, articulando-o com os fatores identificados nesse estudo, percebe-se que esses fatores são como os orifícios do queijo, que quando alinhadas reproduzem um incidente. É fundamental obstruir essas falhas, além de criar diversas camadas de proteção sobrepostas com o objetivo de evitar que os orifícios se alinhem novamente permitindo que o erro aconteça.

Quanto aos fatores clínicos, por serem intrínsecos do paciente, são de difícil controle, entretanto, medidas de prevenção de agravos e promoção de saúde podem ser empregadas.

Sobre o que ocorre após um incidente, $44 \%$ das profissionais afirmaram registro em impresso específico, seguido de registro em prontuário (36\%). Nota-se que o registro em prontuário das informações inerentes ao processo de cuidar é um dever profissional ${ }^{23}$.

Entre as medidas que devem ser adotadas após a ocorrência de incidentes, $48 \%$ opinam a favor de medidas educativas e $32 \%$ sobre a correção de fatores relacionados. Nenhuma enfermeira referiu medidas jurídicas e legais. Ratificando a valorização da qualificação profissional e da interferência do ambiente sobre o trabalho, em detrimento de medidas punitivas isoladas ${ }^{24}$. Assim, diante da ocorrência de um erro deve haver uma análise criteriosa pela Comissão de Ética de Enfermagem e do Núcleo de Segurança do Paciente, objetivando elucidar os fatos, corrigi-los e educar o profissional como medida para evitar a repetição.

Contudo, na literatura, o uso de medidas punitivas, como advertências, suspensões, demissões são frequentes e apenas reiteram a predominância da repreensão na realidade profissional resultando na subnotificação dos incidentes ${ }^{25}$.

Paradoxalmente, apesar da valorização das medidas educativas após incidentes, e da predominância de investimentos em capacitação e treinamento para evitar ocorrência de incidentes, grande parte das enfermeiras não reconhecem deficiências de formação e treinamento como fatores relacionados aos incidentes neste estudo.
Reitera-se que o englobamento de ferramentas teóricas para fundamentar o exercício prático é indispensável para evitar a ocorrência de falhas, além de ser um dever do profissional ${ }^{23}$.

A implantação de uma cultura de segurança foi citada apenas por $28,57 \%$. A cultura de segurança é caracterizada por um comportamento, no qual todos os profissionais envolvidos no cuidado assumem um compromisso com a promoção da segurança. Assim há um estímulo à identificação, notificação e resolução de fatores que interferem nesse quesito, priorizando uma aprendizagem organizacional e ajustando recursos para a promoção da segurança do paciente. 1 Ressalta-se a importância da notificação dos incidentes e uma melhor comunicação entre a equipe.

Melhorias na comunicação estão associadas a prevenção de incidentes, entretanto, ainda é hoje, é possível perceber uma subordinação nas relações de trabalho, o que dificulta uma prática colaborativa.

\section{Conclusão}

A atenção à saúde apresenta riscos devido à complexidade dos sistemas, e assim, os incidentes ocorrem, o que é preocupante principalmente quando se trata de pacientes idosos.

Identificou-se que as enfermeiras afirmam saber sobre a ocorrência de incidentes na prática hospitalar, e que os principais fatores relacionados foram as condições clínicas dos idosos, principalmente a imobilidade, uma das síndromes geriátricas com grande acometimento na funcionalidade e qualidade de vida da pessoa idosa. Dentre os fatores institucionais, o ambiente físico, e entre os humanos, a falta de atenção foram os prevalentes. Quanto aos incidentes mais frequentes, destacam-se o desenvolvimento de lesões por pressão e queda de pacientes. Concluiu-se que os incidentes não são causas, mas, consequências de diversos fatores alinhados.

Garantir a segurança do paciente é um desafio, já que ainda há um abismo entre o cuidado real e o cuidado ideal. Ressalta-se a necessidade de uma análise críti$\mathrm{ca}$, ética e bioética sobre o erro, permitindo identificar os fatores predisponentes, para que estratégias sejam criadas objetivando coibir a recorrência, além de monitorar os riscos potenciais. É indispensável o 
estímulo ao processo de notificação, com a finalidade de melhorar a prática assistencial, a partir da correção das falhas, sem fins punitivos.

Esse estudo aponta a importância da comunicação em equipe e capacitação teórica, além da implantação de protocolos. Como limitação, percebe-se a necessidade de estudos qualitativos para validar e complementar os resultados encontrados, permitindo um aprofundamento da compreensão da segurança.

Sendo assim, considerando o cuidar como exercício profissional da enfermeira, compreender a segurança do paciente é imprescindível para a integralidade desse cuidado. Esse entendimento possibilitará o desenvolvimento de ações e programas de educação permanente com a finalidade de amenizar os fatores intervenientes encontrados, e assim, proporcionar um cuidado especializado e seguro ao idoso hospitalizado.

\section{Contribuição das autoras}

Jesus LA contribuiu na concepção do projeto, coleta, análise e interpretação dos dados, redação do artigo e aprovação da versão final. Medeiros MOSF contribuiu na concepção do projeto, análise e interpretação dos dados e redação do artigo, revisão crítica relevante do conteúdo e aprovação da versão final. Silva MG contribuiu na revisão crítica relevante do conteúdo e aprovação da versão final.

\section{Conflitos de interesses}

Nenhum conflito financeiro, legal ou político envolvendo terceiros (governo, empresas e fundações privadas, etc.) foi declarado para nenhum aspecto do trabalho submetido (incluindo mas não limitando-se a subvenções e financiamentos, participação em conselho consultivo, desenho de estudo, preparação de manuscrito, análise estatística, etc.).

\section{Referências}

1. Brasil. Ministério da Saúde. Portaria MS/ GM n 529, de 1 de abril de 2013 [internet]. Institui o Programa Nacional de Segurança do Paciente (PNSP). Gabinete do Ministro. Brasília, DF: 2013.

2. Gomes ATL, Silva MF, Moraes SHM, Chiavone FBT, Medeiros SM, Santos VEP. Erro humano e cultura de segurança à luz da teoria "queijo suíço": análise reflexiva. Rev Enferm. 2016;10(4):3646-52.
3. Marinho MM, Radunz V, Barbosa SFF. Avaliação da cultura de segurança pelas equipes de enfermagem de unidades cirúrgicas. Texto Contexto Enferm. 2014;23(3):581-90. doi: 10.1590/0104$\underline{07072014002640012}$

4. Brasil. Ministério da Saúde. Documento de referência para o Programa Nacional de Segurança do Paciente. Brasília, DF: Ministério da Saúde; 2014.

5. Organização Mundial de Saúde (OMS). Relatório Mundial de Envelhecimento e Saúde. Genebra: OMS; 2015.

6. Vaccari E, Lenardt MH, Willig MH, Betiolli SE, Andrade LAS. Segurança do paciente idoso e o evento queda no ambiente hospitalar. Cogitare Enferm. 2016; 21(esp):01-09.

7. Ackroyd-Stolarz S, Bowles SK, Giffin L. Validating administrative data for the detection of adverse events in older hospitalized patients. Drug Healthc Patient Saf. 2014;13(6):101-8. doi: 10.2147/ DHPS.S64359

8. Teixeira CC, Bezerra ALQ, Paranaguá TTB, Pagotto V. Prevalência de eventos adversos entre idosos internados em unidade de clínica cirúrgica. Rev Baiana Enferm. 2017;31(3):01-10. doi: $\underline{10.18471 / \text { rbe.v31i3.22079 }}$

9. Toffoletto MC, Barbosa RL, Andolhe R, Oliveira EM, Ducci AJ, Padilha KG. Factors associated with the occurrence of adverse events in critical elderly patients. Rev Bras Enferm. 2016;69(6):97783. doi: 10.1590/0034-7167-2016-0199

10. Tobias GC, Bezerra ALQ, Moreira IA, Paranaguá TTB, Silva AEBC. Knowledge of nurses on the culture of patient safety in university hospital. J Nurs UFPE. 2016;10(3):1071-1079. doi: 10.5205/1981-8963-v10i3a11060p1071-1079-2016

11. Oliveira DU, Ercole FF, Melo LS, Matos SS, Campos CC, Fonseca EAM. Evaluation of falls in hospitalized elderly. J Nurs UFPE. 2017; 11(11):4589-97. doi: 10.5205/1981-8963-v11i11a23119 8p4589-4597-2017

12. Tondo JCA, Guirardello EB. Perception of nursing professionals on patient safety culture. Rev Bras Enferm. 2017;70(6):1355-60. doi: $10.1590 / 0034-7167-2016-0010$

13. Kendall GD, Blegen MA. Competence and certification of registered nurses and safety of patients in intensive care units. Am J Crit Care 2009;18(2):106-13. doi: 10.4037/ajcc2009487

14. Rogers AE, Hwang WT, Scott LD, Aiken LH, Dinges DF. The working hours of hospital staff nurses and patient safety. Health Aff (Millwood) 2004;23(4):202-12. doi: 10.1377/hlthaff.23.4.202

15. Santos JC, Ceolim MF. latrogenias de enfermagem em pacientes idosos hospitalizados. Rev Esc Enferm USP 2009;43(4):810-7. doi: 10.1590/S0080-62342009000400011 
16. Santos CT, Almeida MA, Oliveira MC, Victor MAG, Lucena AF. Development of the nursing diagnosis risk for pressure ulcer. Rev Gaúcha Enferm. 2015;36(2):113-21. doi: 10.1590/1983$\underline{1447.2015 .02 .49102}$

17. Falcão RMM, Costa KNFM, Fernandes MGM, Pontes MLF, Vasconcelos JMB, Oliveira JS. Risco de quedas em pessoas idosas hospitalizadas. Rev Gaúcha Enferm. 2019;40(esp):01-08. doi: 10.1590/1983-1447.2019.20180266

18.Agência Nacional de Vigilância Sanitária (BR). Boletim Informativo. Segurança do paciente e qualidade em serviços de saúde. Brasil. 2011.

19. Milutinović1 D, Simin D, Davor Z. Risk factor for phlebitis: a questionnaire study of nurses' perception. Rev Latino-Am. 2015;23(4):677-84. doi: 10.1590/0104-1169.0192.2603

20. Buzatto LL, Massa GP, Peterlini MAS, Whitaker IY. Fatores relacionados à flebite em idosos com infusão intravenosa de amiodarona. Acta Paul Enferm. 2016; 29(3):260-6. doi: $\underline{10.1590 / 1982-0194201600037}$

21. World Health Organization. Patient Safety: Making health care safer. Geneva: World Health Organization; 2017. [citado em 2019 outubro 01]. Disponível em: https://apps.who.int/iris/bitstream/ handle/10665/255507/WHO-HIS-SDS-2017.11-eng.pdf

22. Novaretti MCZ, Santos EV, Quitério LM, Daud-Gallotti RM. Sobrecarga de trabalho da Enfermagem e incidentes e eventos adversos em pacientes internados em UTI. Rev Bras Enferm 2014; 67(5):692-9. doi: 10.1590/0034-7167.2014670504

23. Conselho Federal de Enfermagem. Resolução n 0564/2017, de 06 de novembro de 2017. Aprova o novo Código de Ética dos Profissionais de Enfermagem.

24. Tavares APM, Moura ECC, Avelino FVSD, Lopes VCAL, Nogueira LT. Patient safety culture from the perspective of the nursing team. Rev. RENE. 2018;19 e3152: 1-8. doi: 10.15253/21756783.2018193152

25. Figueiredo ML, D'Innocenzo M. Eventos adversos relacionados às práticas assistenciais: uma revisão integrativa. Enfermería Global. 2017;47:621-35. doi: 10.6018/eglobal.16.3.256091 